\title{
CONVERGENCE OF REWARD FUNCTIONALS IN A RESELLING MODEL FOR A EUROPEAN OPTION
}

UDC 519.21

\author{
M. S. PUPASHENKO
}

\begin{abstract}
We consider an optimal reselling problem for a European option. A modification of the Cox-Ingersoll-Ross process is used to model the implied volatility. We construct a two-dimensional binomial-trinomial exponential approximation instead of the discrete approximation proposed by Pupashenko and Kukush (2008) in Theory Stoch. Process. 14(30), no. 3-4, 114-128. We use the results concerning the convergence of reward functionals for exponential price processes with independent log-increments obtained by Lundgren et al. (2008) in J. Numer. Appl. Math. 1(96), 90-113. We proved that there is no arbitrage strategy in the proposed discrete model.
\end{abstract}

\section{INTRODUCTION}

We consider a European option. An owner of a European option is not entitled to exercise it before a given maturity time $T$ and should wait until this time. On the other hand, it is known that investors playing in real finance markets are allowed to resell an option prior to the maturity for a quoted market price. One can ask a question about an optimal moment for reselling an option. The optimal reselling problem for a European option is posed in the paper [4].

We use the classical geometric Brownian motion to model the price process of a stock. We assume that the market price of an option is given by the Black-Scholes formula where the implied volatility is used instead of its true value. We also use the main results concerning the convergence of the option rewards for exponential price processes with independent log-increments obtained in [5].

The model of an implied volatility considered in [4] represents the volatility in terms of a geometric Brownian motion. Unfortunately, this model is not acceptable in practice because the implied volatility may differ essentially from its real value as $t$ increases.

Another model based on the Cox-Ingersoll-Ross process is proposed in [6]. However the Cox-Ingersoll-Ross process as well as its discrete approximation proposed in [6] as a model of the implied volatility does not satisfy the assumptions of Theorems 1 and 3 of [5]. Thus we use a modification of a Cox-Ingersoll-Ross process that possesses the necessary properties to model the implied volatility. Note also that we construct an approximation in a different way as compared to that in the paper [6].

The paper is organized as follows. In Section 2 we set the optimal reselling problem. We recall the main results of [5] concerning the convergence of rewards for exponential price processes with independent log-increments in Section 3 . A two-dimensional

2010 Mathematics Subject Classification. Primary 60J05, 60H10; Secondary 91Gxx, 91B70.

Key words and phrases. European option, American option, reselling problem, reward, convergence, optimal stopping time, discrete approximation, Markov process, binomial-trinomial approximation, CoxIngersoll-Ross process. 
binomial-trinomial model is constructed in Section 4. This model approximates the twodimensional diffusion process corresponding to the reselling model. Also, we prove the convergence of optimal expected rewards to the corresponding rewards of the diffusion process in the binomial-trinomial model. We prove that the binomial-trinomial model is arbitrage-free in Section 5 .

\section{Setting of the Reselling PROBlem}

We consider the classical Black-Scholes market for continuous time, namely

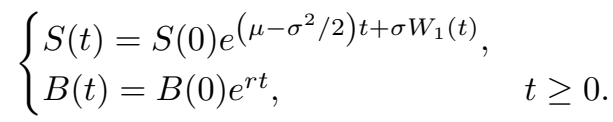

Here $\mu, \sigma$, and $r$ are positive parameters, the values $S(0)$ and $B(0)$ are positive and nonrandom, and $W_{1}(t)$ is a standard Brownian motion adapted to a filtration

$$
\left(\Omega, \mathcal{F},\left(\mathcal{F}^{\prime}{ }_{t}\right)_{t \geq 0}, \mathrm{P}\right) \text {. }
$$

The price of a stock at a moment $t$ for a European option with exercise price $K>0$ and maturity $T>0$ is described by the Black-Scholes formula

$$
C(t, S(t), \sigma)=S(t) \Phi(d)-K e^{-(T-t)} \Phi(d-\sigma \sqrt{T-t}),
$$

where

$$
d=\frac{\ln (S(t) / K)+r(T-t)}{\sigma \sqrt{T-t}}+\frac{\sigma \sqrt{T-t}}{2}, \quad \Phi(x)=\frac{1}{\sqrt{2 \pi}} \int_{-\infty}^{x} e^{-y^{2} / 2} d y .
$$

It is known that a market price of a European option may differ from the theoretical price. Thus a certain stochastic process $\sigma(t)$ appears in formula (2) instead of $\sigma$ to describe the market price. The process $\sigma(t)$ is called an implied volatility.

Consider the following model for $\tilde{\sigma}(t)$ that is based on a Cox-Ingersoll-Ross process (see [3]),

$$
d \widetilde{\sigma}^{2}(t)=-\alpha\left(\widetilde{\sigma}^{2}(t)-\widetilde{\sigma}^{2}\right) d t+\beta \sqrt{\widetilde{\sigma}^{2}(t)} d W_{2}(t), \quad \tilde{\sigma}(0)=\widetilde{\sigma},
$$

where $\alpha, \beta, \widetilde{\sigma}>0, \beta^{2} \leq 2 \alpha \widetilde{\sigma}^{2}$, and where $W_{2}(t)$ also is a standard Brownian motion in the space $\left(\Omega, \mathcal{F},\left(\mathcal{F}^{\prime \prime}{ }_{t}\right)_{t \geq 0}, \mathrm{P}\right)$. The process $W_{2}(t)$ is adapted to another filtration $\left(\mathcal{F}^{\prime \prime}{ }_{t}\right)_{t \geq 0}$. If the above conditions imposed on $\alpha, \beta$, and $\widetilde{\sigma}$ hold, then (3) does not cross the level 0 and thus is positive for $t \geq 0$ in view of $\widetilde{\sigma}>0$.

The mean value and covariance of $\widetilde{\sigma}$ are given by (see, for example, [7]):

$$
\mathrm{E} \widetilde{\sigma}^{2}(t)=\widetilde{\sigma}^{2},
$$

$$
\operatorname{Cov}\left(\tilde{\sigma}^{2}(t), \tilde{\sigma}^{2}(s)\right)=\frac{\tilde{\sigma}^{2} \beta^{2}}{2 \alpha} \cdot e^{-\alpha(s+t)}\left(e^{2 \alpha(s \wedge t)}-1\right),
$$

where $s \wedge t:=\min (s, t)$.

Put $\widetilde{\sigma}=\sqrt{\sigma^{2}-\delta_{0}^{2}}>0$, where $0<\delta_{0}<\sigma$ and the number $\delta_{0}$ is sufficiently small. We model the implied volatility $\sigma(t)$ as follows:

$$
\sigma^{2}(t)=\widetilde{\sigma}^{2}(t)+\delta_{0}^{2}, \quad t \geq 0 .
$$

A motivation for using such a model is that we want to separate $\sigma(t)$ from 0 by a positive constant. As mentioned above, process (3) does not cross the level 0 under the above assumptions imposed on $\alpha, \beta, \sigma$, and $\delta_{0}$ and thus process (6) does not cross this level, too. Then (6) can be rewritten in a different form, namely

$$
\sigma(t)=\sqrt{\widetilde{\sigma}^{2}(t)+\delta_{0}^{2}}, \quad t \geq 0
$$


or, passing to the differential form,

$$
d \sigma^{2}(t)=-\alpha\left(\sigma^{2}(t)-\sigma^{2}\right) d t+\beta \sqrt{\sigma^{2}(t)-\delta_{0}^{2}} d W_{2}(t), \quad \sigma(0)=\sigma .
$$

Note that the stochastic differential equation (8) is well defined in view of the inequality $\sigma(t)>\delta_{0}>0$.

We assume that $\vec{W}(t)=\left(W_{1}(t), W_{2}(t)\right)$ is a two-dimensional Brownian motion whose components are positively correlated, that is,

$$
\mathrm{E} W_{1}(t) W_{2}(t)=\rho t, \quad t \geq 0,
$$

where $\rho \in(0,1]$.

Consider the following stochastic process:

$$
b(t)=\left(\widetilde{\sigma}^{2}(t)-\widetilde{\sigma}^{2}\right) e^{\alpha t}, \quad t \geq 0 .
$$

Then (7) can be rewritten as follows:

$$
\sigma(t)=\sqrt{e^{-\alpha t} b(t)+\sigma^{2}} .
$$

Now we evaluate the cross-covariance function $\mathrm{E} W_{1}(t) \widetilde{\sigma}^{2}(t)$. Using (9), we represent the process $W_{1}(t)$ as follows:

$$
W_{1}(t)=\rho W_{2}(t)+\sqrt{1-\rho^{2}} W_{3}(t),
$$

where $W_{3}(t)$ is a Wiener process that is independent of $W_{2}(t)$.

Applying Itô's formula to the function

$$
\eta(t)=f\left(t, W_{1}(t), \tilde{\sigma}^{2}(t)\right)=W_{1}(t) \cdot \tilde{\sigma}^{2}(t),
$$

we get

$$
\begin{aligned}
d \eta(t)= & d\left(W_{1}(t) \widetilde{\sigma}^{2}(t)\right) \\
= & -\alpha W_{1}(t)\left(\widetilde{\sigma}^{2}(t)-\widetilde{\sigma}^{2}\right) d t+\rho \beta \sqrt{\widetilde{\sigma}^{2}(t)} d t+\left(\rho \widetilde{\sigma}^{2}(t)+\beta W_{1}(t) \sqrt{\widetilde{\sigma}^{2}(t)}\right) d W_{2}(t) \\
& +\sqrt{1-\rho^{2}} \widetilde{\sigma}^{2}(t) d W_{3}(t) .
\end{aligned}
$$

Rewriting the latter equation in the integral form and passing to the expectations, we get the following equation:

$$
\mathrm{E} W_{1}(t) \widetilde{\sigma}^{2}(t)=-\alpha \int_{0}^{t} \mathrm{E} W_{1}(s) \widetilde{\sigma}^{2}(s) d s+\rho \beta \int_{0}^{t} \mathrm{E} \sqrt{\widetilde{\sigma}^{2}(s)} d s .
$$

Here we used the property that the expectation of the Itô integral equals zero.

Differentiating both sides of the latter equality we get a linear differential equation of the first order with respect to the unknown $\mathrm{E} W_{1}(t) \widetilde{\sigma}^{2}(t)$ :

$$
\frac{d}{d t} \mathrm{E} W_{1}(t) \widetilde{\sigma}^{2}(t)=-\alpha \mathrm{E} W_{1}(t) \widetilde{\sigma}^{2}(t)+\rho \beta \mathrm{E} \sqrt{\widetilde{\sigma}^{2}(t)} .
$$

Recalling the initial condition $\mathrm{E} W_{1}(0) \widetilde{\sigma}^{2}(0)=0$ we find a solution of this equation,

$$
\mathrm{E} W_{1}(t) \widetilde{\sigma}^{2}(t)=\rho \beta e^{-\alpha t} \int_{0}^{t} \mathrm{E} \sqrt{\widetilde{\sigma}^{2}(s)} e^{\alpha s} d s=\rho \beta e^{-\alpha t} r(t),
$$

where $r(t)=\int_{0}^{t} \mathrm{E} \sqrt{\widetilde{\sigma}^{2}(s)} e^{\alpha s} d s$.

Hence we derive from equalities (44), (5), and (12) that the following properties of the process $b(t)$ are satisfied:

(1) $\mathrm{E} b(t)=0$,

(2) $\mathrm{E} b^{2}(t)=\frac{\beta^{2} \widetilde{\sigma}^{2}}{2 \alpha}\left(e^{2 \alpha t}-1\right)$,

(3) $\mathrm{E} b(t) b(s)=\frac{\beta^{2} \widetilde{\sigma}^{2}}{2 \alpha}\left(e^{2 \alpha(t \wedge s)}-1\right)$,

(4) $\mathrm{E} W_{1}(t) b(t)=\rho \beta r(t), \rho \in(0,1]$. 
The market price $C(t, S(t), \sigma(t))$ generates a reselling procedure for a European option. We assume that an owner of an option is allowed to resell it at a certain random moment belonging to the class $\mathcal{M}_{T}$ of Markov stopping times $0 \leq \tau \leq T$ with respect to the filtration $\mathcal{F}_{t}=\sigma((S(s), \sigma(s)), s \leq t)$, generated by the vector process $(S(t), \sigma(t))$.

Our aim is to study the reward functional

$$
\Phi\left(\mathcal{M}_{T}\right)=\sup _{\tau \in \mathcal{M}_{T}} \mathrm{E} e^{-r \tau} C(\tau, S(\tau), \sigma(\tau))
$$

Thus the reselling problem of a European option reduces to the optimal exercise problem of an American option with the payoff function $e^{-r t} C(t, S, \sigma)$ for the two-dimensional process $(S(t), \sigma(t))$.

Our approach is based on an approximation of the process $(S(t), \sigma(t))$ with the help of a suitable two-dimensional binomial-trinomial model. Then we solve the following two problems.

The first problem is to construct a binomial-trinomial model that satisfies the corresponding recombination condition (this condition holds for binomial trees if a result of the down-and-up traveling is the same as in the case of the up-and-down traveling). The recombination condition for trinomial trees is defined similarly. The simplest example of a binomial tree that satisfies the recombination condition is presented by the tree $\left\{S_{0}\right\}$, $\left\{S_{0} p, S_{0} q\right\},\left\{S_{0} p^{2}, S_{0} p q, S_{0} q^{2}\right\}, \ldots$. Note that this tree has a polynomial rate of growth of the number of nodes as a function of the number of steps (for example, the growth rate is quadratic for two-dimensional trees).

The second problem is to check the conditions for the convergence of the above reward functionals.

\section{Convergence of ReWARds For exponential PRice processes With INDEPENDENT LOG-INCREMENTS}

In this section, we recall the main results of the paper [5] concerning the convergence of rewards for exponential price processes with independent log-increments.

Given $\varepsilon \geq 0$, let $\vec{Y}^{(\varepsilon)}(t)=\left(Y_{1}^{(\varepsilon)}(t), \ldots, Y_{k}^{(\varepsilon)}(t)\right), t \geq 0$, be a Markov càdlàg process whose phase space is $\mathbb{R}^{k}$. We assume that $\vec{Y}^{(\varepsilon)}(t)$ has independent increments and that the transition probabilities $P^{(\varepsilon)}(t, \vec{y}, t+s, A)$ are related to the distribution of its increments $P^{(\varepsilon)}(t, t+u, A)$ as follows:

$$
P^{(\varepsilon)}(t, \vec{y}, t+u, A)=P^{(\varepsilon)}(t, t+u, A-\vec{y})=\mathrm{P}\left\{\vec{y}+\vec{Y}^{(\varepsilon)}(t+u)-\vec{Y}^{(\varepsilon)}(t) \in A\right\} .
$$

Here $\vec{Y}^{(\varepsilon)}(t)$ is the vector logarithmic price process. For the sake of simplicity, we assume that the initial state $\vec{Y}^{(\varepsilon)}(0)=\vec{y}^{(\varepsilon)}=\left(y_{i}^{(\varepsilon)}, i=1, \ldots, k\right)$ is a nonrandom constant.

Now we define the vector price process $\vec{S}^{(\varepsilon)}(t)=\left(S_{1}^{(\varepsilon)}(t), \ldots, S_{k}^{(\varepsilon)}(t)\right), t \geq 0$, by

$$
S_{i}^{(\varepsilon)}(t)=e^{Y_{i}^{(\varepsilon)}(t)}, \quad i=1, \ldots, k, \quad t \geq 0 .
$$

The phase space of $S_{i}^{(\varepsilon)}$ is $\mathbb{R}_{+}^{k}=\mathbb{R}_{+} \times \cdots \times \mathbb{R}_{+}$, where $\mathbb{R}_{+}:=(0, \infty)$.

Since the exponential function is bijective and continuous, $\vec{S}^{(\varepsilon)}(t)$ is a Markov càdlàg process with independent increments of its logarithm.

Let $\varepsilon \geq 0$ and let $g^{(\varepsilon)}(t, \vec{s}),(t, \vec{s}) \in \mathbb{R}_{+} \times \mathbb{R}_{+}^{k}$, be the payoff function of the corresponding American option. We assume that $g^{(\varepsilon)}(t, \vec{s})$ is a real Borel function.

Let $\mathcal{F}_{t}^{(\varepsilon)}=\sigma\left(\vec{Y}^{(\varepsilon)}(s), s \leq t\right)$ be the natural filtration of $\sigma$-algebras corresponding to the vector logarithmic price process $\vec{Y}^{(\varepsilon)}(t), t \geq 0$. It is worthwhile mentioning that this filtration coincides with the natural filtration generated by the price process $\vec{S}^{(\varepsilon)}(t)$, $t \geq 0$. 
We consider Markov moments $\tau^{(\varepsilon)}$ with respect to the filtration $\mathcal{F}_{t}^{(\varepsilon)}, t \geq 0$. This means that $\tau^{(\varepsilon)}$ is a random variable assuming values in $[0, \infty]$ and such that

$$
\left\{\omega: \tau^{(\varepsilon)}(\omega) \leq t\right\} \in \mathcal{F}_{t}^{(\varepsilon)}, \quad t \geq 0
$$

Let $\mathcal{M}_{\max , T}^{(\varepsilon)}$ be the class of all Markov moments $\tau^{(\varepsilon)} \leq T$, where $T>0$. Consider a subclass of Markov moments $\mathcal{M}_{T}^{(\varepsilon)} \subseteq \mathcal{M}_{\max , T}^{(\varepsilon)}$.

Let $\Pi=\left\{0=t_{0}<t_{1}<\cdots<t_{N}=T\right\}$ be a partition of the interval [0,T] and let $d(\Pi)=\max _{1 \leq i \leq N}\left(t_{i}-t_{i-1}\right)$. By $\mathcal{M}_{\Pi, T}^{(\varepsilon)}$ we denote the class of all Markov times $\tau^{(\varepsilon)}$ of $\mathcal{M}_{\max , T}^{(\varepsilon)}$ assuming values $t_{0}, t_{1}, \ldots, t_{N}$ and such that

$$
\left\{\omega: \tau^{(\varepsilon)}(\omega)=t_{j}\right\} \in \sigma\left(\vec{Y}^{(\varepsilon)}\left(t_{0}\right), \ldots, \vec{Y}^{(\varepsilon)}\left(t_{j}\right)\right)
$$

for $j=0, \ldots, N$.

We are interested in studying the reward functional, that is, the maximum expected gain over various classes of Markov times, namely

$$
\Phi\left(\mathcal{M}_{T}^{(\varepsilon)}\right)=\sup _{\tau^{(\varepsilon)} \in \mathcal{M}_{T}^{(\varepsilon)}} \mathrm{E} g^{(\varepsilon)}\left(\tau^{(\varepsilon)}, \vec{S}^{(\varepsilon)}\left(\tau^{(\varepsilon)}\right)\right) .
$$

In what follows the symbols $\mathrm{E}_{\vec{y}, t}$ and $\mathrm{P}_{\vec{y}, t}$ stand for the conditional expectation and probabilities given condition $\vec{Y}^{(\varepsilon)}(t)=\vec{y}$.

For $\theta, c, T>0$, and $i=1, \ldots, k$ we introduce the modulus of compactness of exponential moments for the càdlàg process $Y_{i}^{(\varepsilon)}(t), t \geq 0$ :

$$
\Delta_{\theta}\left(Y_{i}^{(\varepsilon)}(\cdot), c, T\right)=\sup _{0 \leq t \leq t+u \leq t+c \leq T} \sup _{\vec{y} \in \mathbb{R}_{+}^{k}} \mathrm{E}_{\vec{y}, t}\left(e^{\theta\left|Y_{i}^{(\varepsilon)}(t+u)-Y_{i}^{(\varepsilon)}(t)\right|}-1\right) .
$$

Now we are ready to state Theorems 1 and 3 of the paper [5]. The conditions of these theorems are quite cumbersome and are thus omitted in the statements below. Nevertheless we will check all these conditions carefully when applying these general results.

Theorem 3.1 (see Theorem 1 of [5]). Assume that conditions $\mathbf{A}_{\mathbf{1}}, \mathbf{C}_{\mathbf{1}}$, and $\mathbf{C}_{\mathbf{2}}$ hold. Then there are positive constants $L_{2}, L_{3}<\infty$ such that, for all $\varepsilon \leq \varepsilon_{1}$, one has the following bound for the discrete approximation:

$$
\begin{aligned}
& \Phi\left(\mathcal{M}_{\max , T}^{(\varepsilon)}\right)-\Phi\left(\mathcal{M}_{\Pi, T}^{(\varepsilon)}\right) \\
& \quad \leq L_{2} d(\Pi)+L_{3}\left(\sum_{i=1}^{k} \Delta_{\theta}\left(Y_{i}^{(\varepsilon)}(\cdot), d(\Pi), T\right)\right)^{\frac{\theta-\gamma}{\theta}} .
\end{aligned}
$$

Theorem 3.2 (see Theorem 3 of [5]). Assume that conditions $\mathbf{A}_{\mathbf{1}}, \mathbf{A}_{\mathbf{4}}$, and $\mathbf{D}_{\mathbf{1}}-\mathbf{D}_{\mathbf{4}}$ hold. Then

$$
\Phi\left(\mathcal{M}_{\max , T}^{(\varepsilon)}\right) \rightarrow \Phi\left(\mathcal{M}_{\max , T}^{(0)}\right)<\infty \quad \text { as } \varepsilon \rightarrow 0 .
$$

Note that the assumptions of Theorem 3.2 imply the assumptions of Theorem 3.1 for an arbitrary partition $\Pi$ of the interval $[0, T]$.

\section{THE BINOMIAL-TRINOMIAL APPROXIMATION FOR THE RESELLING MODEL}

We turn to the reselling model introduced in Section 2

Consider the model (11) with $\sigma$ given by (11). Now $(S(t), \sigma(t))$ is a Markov process. We study the reward functional $\Phi\left(\mathcal{M}_{T}\right)$ for an American option with a payoff function $e^{-r t} C(t, S, \sigma)$ for the two-dimensional Markov process $(S(t), \sigma(t))$. 
Consider the following two stochastic processes:

$$
S_{1}^{(0)}(t)=e^{\sigma W_{1}(t)}, \quad t \geq 0, \quad \text { and } \quad S_{2}^{(0)}(t)=e^{b(t)}, \quad t \geq 0 .
$$

Then $\vec{S}^{(0)}(t)=\left(S_{1}^{(0)}(t), S_{2}^{(0)}(t)\right), t \geq 0$, is a two-dimensional continuous nonhomogeneous exponential process with independent log-increments.

It is clear that the filtration $\mathcal{F}_{t}=\sigma((S(s), \sigma(s)), s \leq t)$ generated by the vector process $(S(t), \sigma(t))$ coincides with the filtration $\mathcal{F}_{t}=\sigma\left(\left(S_{1}^{(0)}(s), S_{2}^{(0)}(s)\right), s \leq t\right), t \geq 0$, generated by the process $\vec{S}^{(0)}(t)$.

We introduce the payoff function by

$$
\begin{aligned}
g(t, \vec{s})=e^{-r t} C\left(t, S(0) e^{\left(\mu-\sigma^{2} / 2\right) t} s_{1},\right. & \sqrt{e^{-\alpha t} \ln s_{2}+\sigma^{2}} \\
& \left.\times I_{e^{-\alpha t} \ln s_{2}+\sigma^{2}>\delta_{0}^{2}}+\delta_{0} I_{e^{-\alpha t} \ln s_{2}+\sigma^{2} \leq \delta_{0}^{2}}\right),
\end{aligned}
$$

where the indicator $I_{e^{-\alpha t}} \ln s_{2}+\sigma^{2}>\delta_{0}^{2}$ equals 1 for $\left(t, s_{2}\right)$ such that

$$
e^{-\alpha t} \ln s_{2}+\sigma^{2}>\delta_{0}^{2},
$$

while it equals 0 otherwise.

Note that this payoff function does not depend on the perturbation parameter $\varepsilon$.

Then the reward functional is given by

$$
\Phi\left(\mathcal{M}_{T}\right)=\sup _{\tau \in \mathcal{M}_{T}} \mathrm{E} e^{-r \tau} C(\tau, S(\tau), \sigma(\tau))=\sup _{\tau \in \mathcal{M}_{T}} \mathrm{E} g\left(\tau, \vec{S}^{(0)}(\tau)\right) .
$$

Consider the corresponding two-dimensional logarithmic price process

$$
\vec{Y}^{(0)}(t)=\left(Y_{1}^{(0)}(t), Y_{1}^{(0)}(t)\right), \quad t \geq 0,
$$

with the components

$$
Y_{1}^{(0)}(t)=\sigma W_{1}(t), \quad t \geq 0, \quad \text { and } \quad Y_{2}^{(0)}(t)=b(t), \quad t \geq 0 .
$$

We approximate $\vec{Y}^{(0)}(t)$ by the binomial-trinomial process

$$
\vec{Y}^{(\varepsilon)}(t)=\left(Y_{1}^{(\varepsilon)}(t), Y_{2}^{(\varepsilon)}(t)\right), \quad t \geq 0,
$$

with the components

$$
Y_{i}^{(\varepsilon)}(t)=\sum_{1 \leq n \leq[t / \varepsilon]} Y_{n, i}^{(\varepsilon)}, \quad t \geq 0, \quad i=1,2 .
$$

Here $\varepsilon>0$ and $\vec{Y}_{n}^{(\varepsilon)}=\left(Y_{n, 1}^{(\varepsilon)}, Y_{n, 2}^{(\varepsilon)}\right), n=1,2, \ldots$, are independent random vectors that assume the following six values:

$$
\begin{array}{lll}
\left(+u_{1}^{(\varepsilon)},+u_{2}^{(\varepsilon)}\right), & \left(+u_{1}^{(\varepsilon)}, 0\right), & \left(+u_{1}^{(\varepsilon)},-u_{2}^{(\varepsilon)}\right), \\
\left(-u_{1}^{(\varepsilon)},+u_{2}^{(\varepsilon)}\right), & \left(-u_{1}^{(\varepsilon)}, 0\right), & \left(-u_{1}^{(\varepsilon)},-u_{2}^{(\varepsilon)}\right)
\end{array}
$$

with the probabilities $p_{n,++}^{(\varepsilon)}, p_{n,+}^{(\varepsilon)}, p_{n,+-}^{(\varepsilon)}, p_{n,-+}^{(\varepsilon)}, p_{n,-.}^{(\varepsilon)}$, and $p_{n,--}^{(\varepsilon)}$, respectively, where $u_{2}^{(\varepsilon)} \neq 0$.

For the sake of simplicity, let $\varepsilon=T / N$.

In order to prove the convergence of finite-dimensional distributions of the two-dimensional binomial-trinomial process $\vec{Y}^{(\varepsilon)}(t)$ to the corresponding distributions of the limit two-dimensional process $\vec{Y}^{(0)}(t)$, the expectations, variances, and cross-covariance of the components of the random vector $\left(Y_{n, 1}^{(\varepsilon)}, Y_{n, 2}^{(\varepsilon)}\right)$ and those of the increments

$$
\left(\sigma\left(W_{1}(n \varepsilon)-W_{1}((n-1) \varepsilon)\right), b(n \varepsilon)-b((n-1) \varepsilon)\right)
$$


should coincide for $n=1, \ldots, N$. Note that the values of the vector $\left(Y_{n, 1}^{(\varepsilon)}, Y_{n, 2}^{(\varepsilon)}\right)$ do not depend on $n$ but its distribution does depend on $n$, while the distribution of the increments $\left(\sigma\left(W_{1}(n \varepsilon)-W_{1}((n-1) \varepsilon)\right), b(n \varepsilon)-b((n-1) \varepsilon)\right)$ depends on $n$.

It is important that the jumps $u_{1}^{(\varepsilon)}$ and $u_{2}^{(\varepsilon)}$ are independent of $n$, since the necessary recombination condition holds automatically in this case for the two-dimensional binomial-trinomial tree. The same concerns the necessary quadratic rate of growth of the number of nodes as a function of the number of steps in the tree.

It is clear that

$$
\begin{gathered}
\mathrm{E}\left[\sigma \cdot\left(W_{1}(n \varepsilon)-W_{1}((n-1) \varepsilon)\right)\right]=0, \\
\operatorname{Var}\left[\sigma \cdot\left(W_{1}(n \varepsilon)-W_{1}((n-1) \varepsilon)\right)\right]=\sigma^{2} \varepsilon, \\
\mathrm{E}(b(n \varepsilon)-b((n-1) \varepsilon))=0,
\end{gathered}
$$

and

$$
\begin{aligned}
\sigma_{n, \varepsilon}^{2} & =\operatorname{Var}(b(n \varepsilon)-b((n-1) \varepsilon))=\mathrm{E}(b(n \varepsilon)-b((n-1) \varepsilon))^{2} \\
& =\mathrm{E} b^{2}(n \varepsilon)-2 \mathrm{E} b(n \varepsilon) b((n-1) \varepsilon)+\mathrm{E} b^{2}((n-1) \varepsilon) \\
& =\beta^{2} \widetilde{\sigma}^{2} e^{2 \alpha n \varepsilon} \frac{1-e^{-2 \alpha \varepsilon}}{2 \alpha} .
\end{aligned}
$$

In addition,

$$
\begin{aligned}
\varrho_{n, \varepsilon} & =\mathrm{E}\left[\sigma \cdot\left(W_{1}(n \varepsilon)-W_{1}((n-1) \varepsilon)\right) \cdot(b(n \varepsilon)-b((n-1) \varepsilon))\right] \\
& =\sigma \rho \beta(r(n \varepsilon)-r((n-1) \varepsilon)) .
\end{aligned}
$$

We have to solve the following system of $5 N+1$ equations with $6 N+2$ unknowns:

$$
\left\{\begin{array}{lll}
\mathrm{E}\left[Y_{n, 1}^{(\varepsilon)}\right] & =u_{1}^{(\varepsilon)}\left(2\left(p_{n,++}^{(\varepsilon)}+p_{n,+-}^{(\varepsilon)}+p_{n,+.}^{(\varepsilon)}\right)-1\right) & =0, \\
\operatorname{Var}\left[Y_{n, 1}^{(\varepsilon)}\right] & =\left(u_{1}^{(\varepsilon)}\right)^{2} & =\sigma^{2} \varepsilon, \\
\mathrm{E}\left[Y_{n, 2}^{(\varepsilon)}\right] & =u_{2}^{(\varepsilon)}\left(p_{n,++}^{(\varepsilon)}+p_{n,-+}^{(\varepsilon)}-p_{n,+-}^{(\varepsilon)}-p_{n,--}^{(\varepsilon)}\right) & =0, \\
\operatorname{Var}\left[Y_{n, 2}^{(\varepsilon)}\right] & =\left(u_{2}^{(\varepsilon)}\right)^{2}\left(p_{n,++}^{(\varepsilon)}+p_{n,-+}^{(\varepsilon)}+p_{n,+-}^{(\varepsilon)}+p_{n,--}^{(\varepsilon)}\right) & =\sigma_{n, \varepsilon}^{2} \\
\mathrm{E} Y_{n, 1}^{(\varepsilon)} Y_{n, 2}^{(\varepsilon)} & =u_{1}^{(\varepsilon)} u_{2}^{(\varepsilon)}\left(p_{n,++}^{(\varepsilon)}+p_{n,--}^{(\varepsilon)}-p_{n,-+}^{(\varepsilon)}-p_{++-}^{(\varepsilon)}\right) & =\varrho_{n, \varepsilon}, \\
n=1, \ldots, N . & p_{n,++}^{(\varepsilon)}+p_{n,-+}^{(\varepsilon)}+p_{n,+-}^{(\varepsilon)}+p_{n,--}^{(\varepsilon)}+p_{n,+}^{(\varepsilon)}+p_{n,-}^{(\varepsilon)} & =1,
\end{array}\right.
$$

We search for a solution of the form $u_{2}^{(\varepsilon)}=u \sqrt{\varepsilon}$, where $u \neq 0$ is a parameter to be chosen later (recall that the number of unknowns in this system is larger than the number of equations).

We rewrite the above system as follows:

$$
\begin{cases}u_{1}^{(\varepsilon)} & =\sigma \sqrt{\varepsilon}, \\ u_{2}^{(\varepsilon)} & =u \sqrt{\varepsilon}, \\ p_{n,++}^{(\varepsilon)}+p_{n,+-}^{(\varepsilon)}+p_{n,+\cdot}^{(\varepsilon)} & =\frac{1}{2}, \\ p_{n,++}^{(\varepsilon)}+p_{n,-+}^{(\varepsilon)} & =\frac{\sigma_{n, \varepsilon}^{2}}{2 u^{2} \varepsilon}, \\ p_{n,+-}^{(\varepsilon)}+p_{n,--}^{(\varepsilon)} & =\frac{\sigma_{n, \varepsilon}^{2}}{2 u^{2} \varepsilon}, \\ p_{n,++}^{(\varepsilon)}+p_{n,--}^{(\varepsilon)}-p_{n,-+}^{(\varepsilon)}-p_{+-}^{(\varepsilon)} & =\frac{\varrho_{n, \varepsilon}}{\sigma u \varepsilon}, \\ p_{n,+}^{(\varepsilon)}+p_{n,-}^{(\varepsilon)} & =1-\frac{\sigma_{n, \varepsilon}^{2}}{u^{2} \varepsilon}, \\ n=1, \ldots, N, & \end{cases}
$$


and then in the final form:

$$
\begin{cases}u_{1}^{(\varepsilon)} & =\sigma \sqrt{\varepsilon} \\ u_{2}^{(\varepsilon)} & =u \sqrt{\varepsilon} \\ p_{n,++}^{(\varepsilon)} & =\frac{1}{4}+\frac{\varrho_{n, \varepsilon}}{4 \sigma u \varepsilon}-\frac{1}{2} p_{n,+\cdot}^{(\varepsilon)} \\ p_{n,--}^{(\varepsilon)} & =\frac{\sigma_{n, \varepsilon}^{2}}{2 u^{2} \varepsilon}-\frac{1}{4}-\frac{\varrho_{n, \varepsilon}}{4 \sigma u \varepsilon}+\frac{1}{2} p_{n,+\cdot}^{(\varepsilon)} \\ p_{n,-+}^{(\varepsilon)} & =\frac{\sigma_{n, \varepsilon}^{2}}{2 u^{2} \varepsilon}-\frac{1}{4}-\frac{\varrho_{n, \varepsilon}}{4 \sigma u \varepsilon}+\frac{1}{2} p_{n,+\cdot}^{(\varepsilon)} \\ p_{n,+-}^{(\varepsilon)} & =\frac{1}{4}+\frac{\varrho_{n, \varepsilon}}{4 \sigma u \varepsilon}-\frac{1}{2} p_{n,+\cdot}^{(\varepsilon)} \\ p_{n,-}^{(\varepsilon)} & =1-\frac{\sigma_{n, \varepsilon}^{2}}{u^{2} \varepsilon}-p_{n,+\cdot}^{(\varepsilon)} \\ n=1, \ldots, N . & \end{cases}
$$

Let

$$
p_{n,+}^{(\varepsilon)}=\frac{1}{2}+\frac{\varrho_{n, \varepsilon}}{2 \sigma u \varepsilon}-\frac{\sigma_{n, \varepsilon}^{2}}{u^{2} \varepsilon}
$$

It is easy to show that

$$
\beta^{2} \widetilde{\sigma}^{2} \leq \frac{\sigma_{n, \varepsilon}^{2}}{\varepsilon} \leq \beta^{2} \widetilde{\sigma}^{2} e^{2 \alpha T}
$$

and

$$
0 \leq \frac{\varrho_{n, \varepsilon}}{\varepsilon} \leq \rho \sigma \beta \widetilde{\sigma} e^{\alpha T}
$$

for $n=1, \ldots, N$. Thus we can choose a sufficiently large $u$ such that the above probabilities satisfy the following inequalities:

$$
0<\frac{1}{2}-\frac{\beta^{2} \widetilde{\sigma}^{2} e^{2 \alpha T}}{u^{2}} \leq p_{n,+}^{(\varepsilon)} \leq \frac{1}{2}+\frac{\rho \beta \widetilde{\sigma} e^{\alpha T}}{2 u}-\frac{\beta^{2} \widetilde{\sigma}^{2}}{u^{2}}<1
$$

for $n=1, \ldots, N$.

According to the system of equations (26) we have $p_{n,-+}^{(\varepsilon)}=p_{n,--}^{(\varepsilon)}=0$ if

$$
p_{n,++}^{(\varepsilon)}=p_{n,+-}^{(\varepsilon)}=\frac{\sigma_{n, \varepsilon}^{2}}{2 u^{2} \varepsilon} .
$$

Moreover, the parameter $u$ can be chosen such that

$$
0<\frac{\beta^{2} \widetilde{\sigma}^{2}}{2 u^{2}} \leq p_{n,++}^{(\varepsilon)} \leq \frac{\beta^{2} \widetilde{\sigma}^{2} e^{2 \alpha T}}{2 u^{2}}<1
$$

for all $n=1, \ldots, N$.

Finally

$$
p_{n,-.}^{(\varepsilon)}=1-\frac{\sigma_{n, \varepsilon}^{2}}{u^{2} \varepsilon}-p_{n,+}^{(\varepsilon)}=\frac{1}{2}-\frac{\varrho_{n, \varepsilon}}{2 \sigma u \varepsilon}
$$

according to (26) and $u$ can be chosen such that

$$
0<\frac{1}{2}-\frac{\rho \beta \widetilde{\sigma} e^{\alpha T}}{2 u} \leq p_{n,-}^{(\varepsilon)} \leq \frac{1}{2}<1
$$

for all $n=1, \ldots, N$. 
Thus, if a number $u$ satisfies all the restrictions mentioned above, then the probabilities are given by

$$
\left\{\begin{array}{lll}
p_{n,++}^{(\varepsilon)} & =\frac{\sigma_{n, \varepsilon}^{2}}{2 u^{2} \varepsilon} & =\frac{\beta^{2} \widetilde{\sigma}^{2}}{2 u^{2}} e^{2 \alpha n \varepsilon} \frac{1-e^{-2 \alpha \varepsilon}}{2 \alpha \varepsilon}, \\
p_{n,--}^{(\varepsilon)} & =0 & \\
p_{n,-+}^{(\varepsilon)} & =0 & \\
p_{n,+-}^{(\varepsilon)} & =\frac{\sigma_{n, \varepsilon}^{2}}{2 u^{2} \varepsilon} & =\frac{\beta^{2} \widetilde{\sigma}^{2}}{2 u^{2}} e^{2 \alpha n \varepsilon \frac{1-e^{-2 \alpha \varepsilon}}{2 \alpha \varepsilon}}, \\
p_{n,++}^{(\varepsilon)} & =\frac{1}{2}+\frac{\varrho_{n, \varepsilon}}{2 \sigma u \varepsilon \varepsilon}-\frac{\sigma_{n, \varepsilon}^{2}}{u^{2} \varepsilon} & =\frac{1}{2}+\frac{\rho \beta(r(n \varepsilon)-r((n-1) \varepsilon))}{2 u \varepsilon}-\frac{\beta^{2} \widetilde{\sigma}^{2}}{u^{2}} e^{2 \alpha n \varepsilon} \frac{1-e^{-2 \alpha \varepsilon}}{2 \alpha \varepsilon} \\
p_{n,-}^{(\varepsilon)} & =\frac{1}{2}-\frac{\varrho_{n, \varepsilon}}{2 \sigma u \varepsilon} & =\frac{1}{2}-\frac{\rho \beta(r(n \varepsilon)-r((n-1) \varepsilon))}{2 u \varepsilon} \\
n=1, \ldots, N . &
\end{array}\right.
$$

Further, the moment generating function $\mathrm{E} \exp \left\{\theta\left(Y_{i}^{(\varepsilon)}(t+s)-Y_{i}^{(\varepsilon)}(t)\right)\right\}$ exists for all $\theta \in \mathbb{R}$ and can be evaluated explicitly, namely

$$
\mathrm{E} \exp \left\{\theta\left(Y_{1}^{(\varepsilon)}(t+s)-Y_{1}^{(\varepsilon)}(t)\right)\right\}=\left(e^{\theta u_{1}^{(\varepsilon)}} p_{1,+}^{(\varepsilon)}+e^{-\theta u_{i}^{(\varepsilon)}} p_{1,-}^{(\varepsilon)}\right)^{[(t+s) / \varepsilon]-[t / \varepsilon]}
$$

for $\varepsilon>0$ and $0 \leq t \leq t+s \leq T, i=1,2$. Here

$$
p_{1,+}^{(\varepsilon)}=p_{n,++}^{(\varepsilon)}+p_{n,+-}^{(\varepsilon)}+p_{n,+\cdot}^{(\varepsilon)}, \quad p_{2,-}^{(\varepsilon)}=p_{n,-+}^{(\varepsilon)}+p_{n,--}^{(\varepsilon)}+p_{n,-}^{(\varepsilon)} .
$$

(note that the probabilities $p_{1,+}^{(\varepsilon)}$ and $p_{2,-}^{(\varepsilon)}$ do not depend on $n$ ) and

(28) $\mathrm{E} \exp \left\{\theta\left(Y_{2}^{(\varepsilon)}(t+s)-Y_{2}^{(\varepsilon)}(t)\right)\right\}=\prod_{[t / \varepsilon]+1}^{[(t+s) / \varepsilon]}\left(e^{\theta u_{2}^{(\varepsilon)}} p_{n, 2,+}^{(\varepsilon)}+e^{-\theta u_{2}^{(\varepsilon)}} p_{n, 2,-}^{(\varepsilon)}+p_{n, 2, .}^{(\varepsilon)}\right)$

for $\varepsilon>0$, where

$$
p_{n, 2,+}^{(\varepsilon)}=p_{n,++}^{(\varepsilon)}+p_{n,-+}^{(\varepsilon)}, \quad p_{n, 2,-}^{(\varepsilon)}=p_{n,+-}^{(\varepsilon)}+p_{n,--}^{(\varepsilon)}, \quad p_{n, 2,+}^{(\varepsilon)}=p_{n,+\cdot}^{(\varepsilon)}+p_{n,-\cdot}^{(\varepsilon)} .
$$

Now we check the assumptions of Theorems 3.1 and 3.2. As mentioned in Section 3 , we have to check conditions $\mathbf{A}_{\mathbf{1}}, \mathbf{A}_{\mathbf{4}}$ and $\mathbf{D}_{\mathbf{1}}-\mathbf{D}_{\mathbf{4}}$.

Recall that payoff function (20) does not depend on $\varepsilon$ and thus condition $\mathbf{A}_{\mathbf{4}}$ holds automatically.

Next we check condition $\mathbf{A}_{\mathbf{1}}$ :

(a) the function $g(t, \vec{s})$ is absolutely continuous in $t$ with respect to the Lebesgue measure on $[0, T]$ for all fixed $\vec{s} \in \mathbb{R}_{+}^{2}$; also it is absolutely continuous in $\vec{s}$ with respect to the Lebesgue measure on $\mathbb{R}_{+}^{2}$ for all fixed $t \in[0, T]$;

(b) for all $\vec{s} \in \mathbb{R}_{+}^{2}$, the partial derivative $\frac{\partial g}{\partial t}$ is such that

$$
\begin{aligned}
\left|\frac{\partial g(t, \vec{s})}{\partial t}\right| & =\mid-r g(t, \vec{s})+e^{-r t}\left(C_{1}^{\prime}+C_{2}^{\prime} S(0)\left(\mu-\frac{\sigma^{2}}{2}\right) e^{\left(\mu-\sigma^{2} / 2\right) t} s_{1}\right. \\
& \left.\quad+C_{3}^{\prime} \frac{-\alpha e^{-\alpha t} \ln s_{2}}{2 \sqrt{e^{-\alpha t} \ln s_{2}+\sigma^{2}}} I_{e^{-\alpha t} \ln s_{2}+\sigma^{2}>\delta_{0}^{2}}\left(t, s_{2}\right)\right) \mid \\
\leq &
\end{aligned}
$$

for almost all $t \in[0, T]$ with respect to the Lebesgue measure on the interval $[0, T]$ and for some $0 \leq K_{1}, K_{2}<\infty$. Here $C_{1}^{\prime}, C_{2}^{\prime}$, and $C_{3}^{\prime}$ are the partial derivatives with respect to the first, second, and third argument, respectively; 
(c) for all $t \in[0, T]$,

$$
\begin{gathered}
\left|\frac{\partial g^{(\varepsilon)}(t, \vec{s})}{\partial s_{1}}\right|=\left|e^{-r t} C_{2}^{\prime} S(0) e^{\left(\mu-\sigma^{2} / 2\right) t}\right| \leq K_{3}, \\
\left|\frac{\partial g^{(\varepsilon)}(t, \vec{s})}{\partial s_{2}}\right|=\left|e^{-r t} C_{3}^{\prime} \frac{e^{-\alpha t} s_{2}^{-1}}{2 \sqrt{e^{-\alpha t} \ln s_{2}+\sigma^{2}}} I_{e^{-\alpha t} \ln s_{2}+\sigma^{2}>\delta_{0}^{2}}\right| \leq K_{4}
\end{gathered}
$$

for almost all $\vec{s} \in \mathbb{R}_{+}^{2}$ with respect to the Lebesgue measure on $\mathbb{R}_{+}^{2}$ and for some $0 \leq K_{3}, K_{4}<\infty$

(d) for all $t \in[0, T]$ and some $0 \leq K_{5}<\infty$,

$$
g(t, \overrightarrow{0})=\varlimsup_{\vec{s} \rightarrow \overrightarrow{0}} g(t, \vec{s})=e^{-r t} C\left(t, 0, \delta_{0}\right) \leq K_{5} .
$$

Thus condition $\mathbf{A}_{\mathbf{1}}$ holds for the payoff function (20) with some constants $K_{i}, i=1, \ldots, 5$, and with parameters $\gamma_{0}=1$ and $\gamma_{1}=\gamma_{2}=0$, whence $\gamma=\max \left\{\gamma_{0}, \gamma_{1}, \gamma_{2}\right\}=1$.

Note that we use model (7) instead of (3) in order to have $\sigma(t)$ separated from zero by a positive constant $\delta_{0}$, since all the restrictions involved in condition $\mathbf{A}_{\mathbf{1}}$ hold in this case.

Applying the corresponding limit theorems for the vector sum process with independent increments (see [2], §34), one can check that the processes $\vec{Y}^{(\varepsilon)}(t), t \in[0, T]$, with parameters given by (26) weakly converge in the Skorokhod space to the process $\vec{Y}^{(0)}(t)$, $t \in[0, T]$, as $\varepsilon \rightarrow 0$. Thus condition $\mathbf{D}_{\mathbf{1}}$ holds.

The distribution of the sum process (23) is given by (26). Condition $\mathbf{D}_{\mathbf{2}}$ holds for this distribution. Further, by using relations (27) and (28) one can easily check condition $\mathbf{D}_{\mathbf{3}}$ for the processes $\vec{Y}^{(\varepsilon)}(t)$ for all $\theta^{\prime}>\theta$.

Note also that conditions $\mathbf{D}_{\mathbf{1}}$ and $\mathbf{D}_{\mathbf{2}}$ imply J-convergence of the processes $\vec{Y}^{(\varepsilon)}(t)$, $t \in[0, T]$, to the process $\vec{Y}^{(0)}(t), t \in[0, T]$, as $\varepsilon \rightarrow 0$ (see [2], §39).

Finally, condition $\mathbf{D}_{4}$ automatically holds, since the value $\vec{Y}^{(\varepsilon)}(0)=(0,0)$ does not depend on $\varepsilon$.

Summarizing we conclude that all the assumptions of Theorem 3.2 are satisfied, whence we derive that $\Phi\left(\mathcal{M}_{\max , T}^{(\varepsilon)}\right) \rightarrow \Phi\left(\mathcal{M}_{\max , T}^{(0)}\right)$ as $\varepsilon \rightarrow 0$ for the corresponding twodimensional exponential price processes $\vec{S}^{(\varepsilon)}(t)=\exp \left\{\vec{Y}^{(\varepsilon)}(t)\right\}, t \geq 0$, with independent log-increments.

Now we consider a partition

$$
\Pi_{\varepsilon}=\left\langle t_{0}=0<t_{1}=\varepsilon<\cdots<t_{N-1}=(N-1) \varepsilon<t_{N}=T\right\rangle
$$

of the interval $[0, T]$.

The Markov chain $\left(n, \vec{Y}^{(\varepsilon)}(n \varepsilon)\right), n \geq 0$, is a binomial-trinomial tree in this case with the initial node $(0,(0,0))$ and with $(n+1)(2 n+1)$ nodes of the form

$$
\left(n,\left(\left(2 l_{1}-n\right) \sqrt{\varepsilon} \sigma, l_{2} \sqrt{\varepsilon} u\right)\right), \quad l_{1}=0, \ldots, n, \quad l_{2}=-n, \ldots, n, \quad i=1,2,
$$

after $n \geq 1$ steps.

If a continuous model of an option with maturity $T$ is approximated by the corresponding discrete model with step $\varepsilon=T / N$, then the corresponding tree has $N$ levels with $(N+1)(2 N+1)$ nodes at the last $N$-th level, and with $((N-1)+1)(2(N-1)+1)$ nodes at the $(N-1)$-th level.

The standard backward procedure can be used to find an optimal expected reward at the moment 0 for the discrete exponential two-dimensional binomial-trinomial price process $\vec{S}^{(\varepsilon)}(n \varepsilon)=\exp \left\{\vec{Y}^{(\varepsilon)}(n \varepsilon)\right\}$. This optimal expected reward coincides in this case with the reward functional $\Phi\left(\mathcal{M}_{\Pi_{\varepsilon}, T}^{(\varepsilon)}\right)$ for the two-dimensional exponential price processes $\vec{S}(\varepsilon)(t)=e^{\vec{Y}^{(\varepsilon)}(t)}$. 
To obtain a bound for the difference $\Phi\left(\mathcal{M}_{\max , T}^{(\varepsilon)}\right)-\Phi\left(\mathcal{M}_{\Pi_{\varepsilon}, T}^{(\varepsilon)}\right)$ we use Theorem 3.1 . In this case, $d\left(\Pi_{\varepsilon}\right)=\varepsilon, \Delta_{\theta}\left(Y_{1}^{(\varepsilon)}(\cdot), \varepsilon, T\right)=\mathrm{E} e^{\theta\left|Y_{1,1}^{(\varepsilon)}\right|}-1 \leq e^{\theta \sigma \sqrt{\varepsilon}}-1$, and

$$
\Delta_{\theta}\left(Y_{2}^{(\varepsilon)}(\cdot), \varepsilon, T\right)=\max _{1 \leq n \leq N}\left(\mathrm{E} e^{\theta\left|Y_{n, 2}^{(\varepsilon)}\right|}-1\right) \leq e^{\theta u \sqrt{\varepsilon}}-1 .
$$

Theorem 3.1 gives in this case

$$
\begin{aligned}
& \Phi\left(\mathcal{M}_{\max , T}^{(\varepsilon)}\right)-\Phi\left(\mathcal{M}_{\Pi_{\varepsilon}, T}^{(\varepsilon)}\right) \\
& \quad \leq L_{2} \varepsilon+L_{3}\left(e^{\theta \sigma \sqrt{\varepsilon}}-1+e^{\theta u \sqrt{\varepsilon}}-1\right)^{\frac{\theta-\gamma}{\theta}} \rightarrow 0 \quad \text { as } \varepsilon \rightarrow 0 .
\end{aligned}
$$

Now Theorem 3.2 implies that the optimal expected reward $\Phi\left(\mathcal{M}_{\Pi_{\varepsilon}, T}^{(\varepsilon)}\right)$ converges to the reward functional $\Phi\left(\mathcal{M}_{\max , T}^{(0)}\right)$ for the two-dimensional process $\vec{S}^{(0)}(t)=e^{\vec{Y}^{(0)}(t)}$ with independent log-increments.

According to Theorem 3.2 the optimal expected reward for the two-dimensional binomial-trinomial exponential model described above converges to the optimal expected reward functional for the corresponding two-dimensional process with independent logincrements.

\section{The Discrete MOdel is ARBitRAGE-FREE}

We start with a classical definition of the arbitrage strategy (see [1]):

Definition 5.1. We say that a Markov stopping time $\tau$ admits an arbitrage if the following two conditions hold:

a) $\mathrm{P}\left(e^{-r \tau} C(\tau, S(\tau), \sigma(\tau)) \geq C(0, S(0), \sigma)\right)=1$,

b) $\mathrm{P}\left(e^{-r \tau} C(\tau, S(\tau), \sigma(\tau))>C(0, S(0), \sigma)\right)>0$.

Consider a partition

$$
\Pi_{\varepsilon}=\left\langle t_{0}=0<t_{1}=\varepsilon<\cdots<t_{N-1}=(N-1) \varepsilon<t_{N}=T\right\rangle
$$

of the interval $[0, T]$. We again use the approximation (22), (23) for $\sigma W_{1}(t), t \geq 0$, and $b(t), t \geq 0$. Using (11) and (11), one can write down an approximation for $S(t)$ and $\sigma(t)$ as follows:

$$
\begin{cases}S^{(\varepsilon)}(t) & =S^{(\varepsilon)}\left(t_{j}\right), \quad t \in\left[t_{j}, t_{j+1}\right), S^{(\varepsilon)}(0)=S(0), \\ \sigma^{(\varepsilon)}(t) & =\sigma^{(\varepsilon)}\left(t_{j}\right), \quad t \in\left[t_{j}, t_{j+1}\right), \sigma^{(\varepsilon)}(0)=\sigma, \\ S^{(\varepsilon)}\left(t_{j+1}\right) & =S^{(\varepsilon)}\left(t_{j}\right) e^{\left(\mu-\sigma^{2} / 2\right) \varepsilon+Y_{j+1,1}^{(\varepsilon)},} \\ \sigma^{(\varepsilon)}\left(t_{j+1}\right) & =\sqrt{e^{-\alpha \varepsilon}\left(\left(\sigma^{(\varepsilon)}\left(t_{j}\right)\right)^{2}+e^{-\alpha t_{j}} Y_{j+1,2}^{(\varepsilon)}\right)+\sigma^{2}\left(1-e^{-\alpha \varepsilon}\right)}, \quad j=0, \ldots, N-1 .\end{cases}
$$

If a martingale measure $P^{*}$ exists for which $e^{-r t_{j}} C\left(t_{j}, S^{(\varepsilon)}\left(t_{j}\right), \sigma^{(\varepsilon)}\left(t_{j}\right)\right)$ is a martingale with respect to the filtration generated by the vector $\left(S^{(\varepsilon)}\left(t_{j}\right), \sigma^{(\varepsilon)}\left(t_{j}\right)\right), j=0, \ldots, N$, then the model is arbitrage-free (see [1]). The latter condition holds if, for all $1 \leq j \leq N$,

$$
\begin{gathered}
\mathrm{E}^{*}\left(e^{-r t_{j}} C\left(t_{j}, S^{(\varepsilon)}\left(t_{j}\right), \sigma^{(\varepsilon)}\left(t_{j}\right)\right) \mid S^{(\varepsilon)}\left(t_{j-1}\right), \sigma^{(\varepsilon)}\left(t_{j-1}\right)\right) \\
=e^{-r t_{j-1}} C\left(t_{j-1}, S^{(\varepsilon)}\left(t_{j-1}\right), \sigma^{(\varepsilon)}\left(t_{j-1}\right)\right) .
\end{gathered}
$$

The symbol $E^{*}$ in equality (30) stands for the expectation with respect to the martingale measure $P^{*}$. 
We choose a measure $P^{*}$ such that $Y_{j, 1}^{(\varepsilon)}$ and $Y_{j, 2}^{(\varepsilon)}$ are independent and have the following distributions:

$$
\begin{aligned}
P^{*}\left(Y_{j, 1}^{(\varepsilon)}=\sigma \sqrt{\varepsilon}\right) & =\frac{e^{r \varepsilon-\left(\mu-\sigma^{2} / 2\right) \varepsilon}-e^{-\sigma \sqrt{\varepsilon}}}{e^{\sigma \sqrt{\varepsilon}}-e^{-\sigma \sqrt{\varepsilon}}}, \\
P^{*}\left(Y_{j, 1}^{(\varepsilon)}=-\sigma \sqrt{\varepsilon}\right) & =1-P^{*}\left(Y_{j, 1}^{(\varepsilon)}=\sigma \sqrt{\varepsilon}\right), \\
P^{*}\left(Y_{j, 2}^{(\varepsilon)}=u \sqrt{\varepsilon}\right) & =h\left(S^{(\varepsilon)}\left(t_{j-1}\right), \sigma^{(\varepsilon)}\left(t_{j-1}\right)\right), \\
P^{*}\left(Y_{j, 2}^{(\varepsilon)}=-u \sqrt{\varepsilon}\right) & =g\left(S^{(\varepsilon)}\left(t_{j-1}\right), \sigma^{(\varepsilon)}\left(t_{j-1}\right)\right), \\
P^{*}\left(Y_{j, 2}^{(\varepsilon)}=0\right) & =f\left(S^{(\varepsilon)}\left(t_{j-1}\right), \sigma^{(\varepsilon)}\left(t_{j-1}\right)\right), \quad j=1, \ldots, N, \\
f\left(S^{(\varepsilon)}\left(t_{j-1}\right), \sigma^{(\varepsilon)}\left(t_{j-1}\right)\right) & =1-h\left(S^{(\varepsilon)}\left(t_{j-1}\right), \sigma^{(\varepsilon)}\left(t_{j-1}\right)\right)-g\left(S^{(\varepsilon)}\left(t_{j-1}\right), \sigma^{(\varepsilon)}\left(t_{j-1}\right)\right) .
\end{aligned}
$$

Introducing the notation

$$
\begin{gathered}
\sigma_{j+1,+}=\sigma_{j+1,+}\left(\sigma^{(\varepsilon)}\left(t_{j}\right)\right)=\sqrt{e^{-\alpha \varepsilon}\left(\left(\sigma^{(\varepsilon)}\left(t_{j}\right)\right)^{2}+e^{-\alpha t_{j}} u \sqrt{\varepsilon}\right)+\sigma^{2}\left(1-e^{-\alpha \varepsilon}\right)}, \\
\sigma_{j+1,-}=\sigma_{j+1,-}\left(\sigma^{(\varepsilon)}\left(t_{j}\right)\right)=\sqrt{e^{-\alpha \varepsilon}\left(\left(\sigma^{(\varepsilon)}\left(t_{j}\right)\right)^{2}-e^{-\alpha t_{j}} u \sqrt{\varepsilon}\right)+\sigma^{2}\left(1-e^{-\alpha \varepsilon}\right)}, \\
\sigma_{j+1,0}=\sigma_{j+1,0}\left(\sigma^{(\varepsilon)}\left(t_{j}\right)\right)=\sqrt{e^{-\alpha \varepsilon}\left(\sigma^{(\varepsilon)}\left(t_{j}\right)\right)^{2}+\sigma^{2}\left(1-e^{-\alpha \varepsilon}\right)}, \quad j=0, \ldots, N-1,
\end{gathered}
$$

we obtain

$$
\begin{aligned}
& \mathrm{E}^{*}\left(e^{-r t_{j}} C\left(t_{j}, S^{(\varepsilon)}\left(t_{j}\right), \sigma^{(\varepsilon)}\left(t_{j}\right)\right) \mid S^{(\varepsilon)}\left(t_{j-1}\right), \sigma^{(\varepsilon)}\left(t_{j-1}\right)\right) \\
&=e^{-r t_{j} \mathrm{E}^{*}[} C\left(t_{j}, S^{(\varepsilon)}\left(t_{j}\right), \sigma_{j,+}\right) h\left(S^{(\varepsilon)}\left(t_{j-1}\right), \sigma^{(\varepsilon)}\left(t_{j-1}\right)\right) \\
&+C\left(t_{j}, S^{(\varepsilon)}\left(t_{j}\right), \sigma_{j,-}\right) g\left(S^{(\varepsilon)}\left(t_{j-1}\right), \sigma^{(\varepsilon)}\left(t_{j-1}\right)\right) \\
&\left.+C\left(t_{j}, S^{(\varepsilon)}\left(t_{j}\right), \sigma_{j, 0}\right) f\left(S^{(\varepsilon)}\left(t_{j-1}\right), \sigma^{(\varepsilon)}\left(t_{j-1}\right)\right) \mid S^{(\varepsilon)}\left(t_{j-1}\right), \sigma^{(\varepsilon)}\left(t_{j-1}\right)\right] \\
&=e^{-r t_{j-1}}[ C\left(t_{j-1}, S^{(\varepsilon)}\left(t_{j-1}\right), \sigma_{j,+}\right) h\left(S^{(\varepsilon)}\left(t_{j-1}\right), \sigma^{(\varepsilon)}\left(t_{j-1}\right)\right) \\
&+C\left(t_{j-1}, S^{(\varepsilon)}\left(t_{j-1}\right), \sigma_{j,-}\right) g\left(S^{(\varepsilon)}\left(t_{j-1}\right), \sigma^{(\varepsilon)}\left(t_{j-1}\right)\right) \\
&\left.+C\left(t_{j-1}, S^{(\varepsilon)}\left(t_{j-1}\right), \sigma_{j, 0}\right) f\left(S^{(\varepsilon)}\left(t_{j-1}\right), \sigma^{(\varepsilon)}\left(t_{j-1}\right)\right)\right] .
\end{aligned}
$$

Now we rewrite (30) as follows:

$$
\begin{aligned}
C\left(t_{j-1},\right. & \left.S^{(\varepsilon)}\left(t_{j-1}\right), \sigma_{j,+}\right) h\left(S^{(\varepsilon)}\left(t_{j-1}\right), \sigma^{(\varepsilon)}\left(t_{j-1}\right)\right) \\
& +C\left(t_{j-1}, S^{(\varepsilon)}\left(t_{j-1}\right), \sigma_{j,-}\right) g\left(S^{(\varepsilon)}\left(t_{j-1}\right), \sigma^{(\varepsilon)}\left(t_{j-1}\right)\right) \\
& +C\left(t_{j-1}, S^{(\varepsilon)}\left(t_{j-1}\right), \sigma_{j, 0}\right) f\left(S^{(\varepsilon)}\left(t_{j-1}\right), \sigma^{(\varepsilon)}\left(t_{j-1}\right)\right) \\
= & C\left(t_{j-1}, S^{(\varepsilon)}\left(t_{j-1}\right), \sigma^{(\varepsilon)}\left(t_{j-1}\right)\right),
\end{aligned}
$$

which is equivalent to

$$
\begin{aligned}
{\left[C_{j-1,+}\right.} & \left.-C_{j-1,0}\right] h\left(S^{(\varepsilon)}\left(t_{j-1}\right), \sigma^{(\varepsilon)}\left(t_{j-1}\right)\right) \\
& +\left[C_{j-1,-}-C_{j-1,0}\right] g\left(S^{(\varepsilon)}\left(t_{j-1}\right), \sigma^{(\varepsilon)}\left(t_{j-1}\right)\right) \\
= & C_{j-1}-C_{j-1,0}
\end{aligned}
$$


where

$$
\begin{aligned}
C_{j-1,+} & =C\left(t_{j-1}, S^{(\varepsilon)}\left(t_{j-1}\right), \sigma_{j,+}\right), \\
C_{j-1,-} & =C\left(t_{j-1}, S^{(\varepsilon)}\left(t_{j-1}\right), \sigma_{j,-}\right), \\
C_{j-1,0} & =C\left(t_{j-1}, S^{(\varepsilon)}\left(t_{j-1}\right), \sigma_{j, 0}\right), \\
C_{j-1} & =C\left(t_{j-1}, S^{(\varepsilon)}\left(t_{j-1}\right), \sigma^{(\varepsilon)}\left(t_{j-1}\right)\right) .
\end{aligned}
$$

If $\varepsilon$ is sufficiently small, then

$$
\begin{aligned}
& \sigma_{j,-}<\sigma_{j, 0}<\sigma^{(\varepsilon)}\left(t_{j-1}\right)<\sigma_{j,+} \text { if } \sigma<\sigma^{(\varepsilon)}\left(t_{j-1}\right), \\
& \sigma_{j,-}<\sigma^{(\varepsilon)}\left(t_{j-1}\right)<\sigma_{j, 0}<\sigma_{j,+} \text { if } \sigma>\sigma^{(\varepsilon)}\left(t_{j-1}\right), \\
& \sigma_{j,-}<\sigma_{j, 0}=\sigma^{(\varepsilon)}\left(t_{j-1}\right)<\sigma_{j,+} \text { if } \sigma=\sigma^{(\varepsilon)}\left(t_{j-1}\right)
\end{aligned}
$$

for all $1 \leq j \leq N$.

Since $C$ is increasing with respect to the third argument, the following inequalities hold if $\varepsilon$ is sufficiently small:

$$
\begin{array}{ll}
C_{j-1,-}<C_{j-1,0}<C_{j-1}<C_{j-1,+} & \text { if } \sigma<\sigma^{(\varepsilon)}\left(t_{j-1}\right), \\
C_{j-1,-}<C_{j-1}<C_{j-1,0}<C_{j-1,+} & \text { if } \sigma>\sigma^{(\varepsilon)}\left(t_{j-1}\right), \\
C_{j-1,-}<C_{j-1,0}=C_{j-1}<C_{j-1,+} & \text { if } \sigma=\sigma^{(\varepsilon)}\left(t_{j-1}\right)
\end{array}
$$

for all $1 \leq j \leq N$.

These inequalities allow us to choose the necessary probabilities $h, g$, and $f$ such that they are nonzero and relation (31) holds. Note that the expression involving these probabilities depends on specific relations between $\sigma$ and $\sigma^{(\varepsilon)}\left(t_{j-1}\right)$. Consider, for example, the case of $\sigma<\sigma^{(\varepsilon)}\left(t_{j-1}\right)$. Then

$$
C_{j-1,+}-C_{j-1,0}>C_{j-1}-C_{j-1,0}>0 \quad \text { and } \quad C_{j-1,-}-C_{j-1,0}<0 .
$$

Rewriting (31) as

$$
\begin{aligned}
& h\left(S^{(\varepsilon)}\left(t_{j-1}\right), \sigma^{(\varepsilon)}\left(t_{j-1}\right)\right)+\frac{C_{j-1,-}-C_{j-1,0}}{C_{j-1,+}-C_{j-1,0}} g\left(S^{(\varepsilon)}\left(t_{j-1}\right), \sigma^{(\varepsilon)}\left(t_{j-1}\right)\right) \\
& \quad=\frac{C_{j-1}-C_{j-1,0}}{C_{j-1,+}-C_{j-1,0}},
\end{aligned}
$$

we find the probabilities $h, g$, and $f$ such that none of them equals zero.

Thus we proved that the above discrete model is arbitrage-free.

\section{CONCLUding REMARKS}

In this paper, we considered the reselling problem of a European option for the case where the implied volatility is described with the help of a modified Cox-Ingersoll-Ross process. For this model, we constructed a two-dimensional binomial-trinomial exponential approximation and proved that it is arbitrage-free. Also we proved the convergence of the optimal expected reward in the model to the optimal expected reward functional for the corresponding two-dimensional exponential process with independent log-increments. A nonmodified Cox-Ingersoll-Ross process as a model of the implied volatility can also be studied in the context of the same problem.

\section{ACKNowledGment}

The author is grateful to Professor O. G. Kukush for support and fruitful discussions concerning the results of the paper. 


\section{BIBLIOGRAPHY}

1. M. M. Leonenko, Yu. S. Mishura, V. M. Parkhomenko, and M. I. Yadrenko, ProbabilityTheoretical and Statistical Methods in Economics and Finance Mathematics, Informtekhnika, Kyiv, 1995. (Ukrainian)

2. A. V. Skorokhod, Random Processes with Independent Increments, Nauka, Moscow, 1964; English transl., Kluwer Academic Publishers Group, Dordrecht, 1991. MR1155400 (93a:60114)

3. J. Cox, J. Ingersoll, and S. Ross, A theory of the term structure of interest rates, Econometrica 53 (1985), 385-407. MR785475

4. A. G. Kukush, Yu. S. Mishura, and G. M. Shevchenko, On reselling of European option, Theory Stoch. Process. 12(28) (2006), 75-87. MR2316567 (2008e:62171)

5. R. Lundgren, D. Silvestrov, and A. Kukush, Reselling of options and convergence of option rewards, J. Numer. Appl. Math. 1(96) (2008), 90-113.

6. M. Pupashenko and A. Kukush, Reselling of European option if the implied volatility varies as Cox-Ingersoll-Ross process, Theory Stoch. Process. 14(30) (2008), no. 3-4, 114-128. MR2498609 (2010h:62318)

7. S. E. Shreve, Lectures on Stochastic Calculus and Finance, Springer, New York, 1997.

Department of Probability Theory, Statistics, and Actuarial Mathematics, Faculty for Mechanics and Mathematics, National Taras Shevchenko University, Academician Glushkov Avenue 2, Kiev 03127, Ukraine

E-mail address: myhailo.pupashenko@gmail.com

Received 22/APR/2010

Translated by S. KVASKO 